livraisons

d'Histoire

de l'Architecture

\section{Livraisons de l'histoire de l'architecture}

13 | 2007

Architectures des établissements d'enseignement supérieur

\title{
Un « Port-Royal laïque » : l'École normale supérieure d'institutrices, à Fontenay-aux-Roses
}

A 'secular Port-Royal' : the Ecole normale supérieure for schoolmistresses in Fontenay-aux-Roses

Die École normale supérieure d'institutrices von Fontenay-aux-Roses : eine laizistische Frauenhochschule

Marc Le Cœur

\section{OpenEdition}

Journals

Édition électronique

URL : http://journals.openedition.org/lha/405

DOI : $10.4000 /$ lha. 405

ISSN : 1960-5994

Éditeur

Association Livraisons d'histoire de l'architecture - LHA

Édition imprimée

Date de publication : 10 juin 2007

Pagination : 65-76

ISSN : 1627-4970

Référence électronique

Marc Le Cœur, « Un « Port-Royal laïque » : l'École normale supérieure d'institutrices, à Fontenay-auxRoses », Livraisons de l'histoire de l'architecture [En ligne], 13 | 2007, mis en ligne le 10 juin 2009. consulté le 30 avril 2019. URL : http://journals.openedition.org/lha/405 ; DOI : 10.4000//ha.405

Ce document a été généré automatiquement le 30 avril 2019

Tous droits réservés à l'Association LHA 


\title{
Un « Port-Royal laïque » : l’École normale supérieure d'institutrices, à Fontenay-aux-Roses
}

\author{
A 'secular Port-Royal' : the Ecole normale supérieure for schoolmistresses in \\ Fontenay-aux-Roses \\ Die École normale supérieure d'institutrices von Fontenay-aux-Roses : eine \\ laizistische Frauenhochschule
}

Marc Le Cœur

1 L'École normale supérieure d'institutrices tient une place particulière dans la geste républicaine. Là, pour la première fois en effet, « l'État appliquait toutes ses ressources, et l'Université les forces réunies de ses trois ordres d'enseignement, à fonder en vue des écoles populaires l'instruction supérieure des jeunes filles $»^{1}$. Instrument de l'émancipation des femmes en même temps que pièce maîtresse de l'enseignement primaire, l'École ne s'incarna pourtant pas dans des constructions monumentales, à l'instar de la nouvelle Sorbonne ou d'autres établissements d'instruction contemporains². La réputation de cet établissement dont on se plaisait à dire qu'il était « unique au monde $\|^{3}$ tranchait avec la retenue de son architecture. Ce paradoxe apparent procède d'abord d'une histoire matérielle jalonnée de déconvenues et de projets avortés; il tient aussi à ce que les bâtiments furent édifiés au gré des besoins et sans plan d'ensemble préalable; il tient enfin et surtout à ce que l'architecte dut se conformer à « l'esprit de Fontenay ", fait de probité laïque et de gravité, autant que de simplicité, de douceur et de gaietét.

\section{Une genèse précipitée et incertaine}

Lorsque Jules Ferry fut nommé ministre de l'instruction publique, le 4 février 1879, la France ne comptait que 79 écoles normales d'instituteurs et 19 écoles normales d'institutrices, dont certaines étaient encore aux mains de congrégations ${ }^{5}$. Avant même 
de songer à étendre les bénéfices de l'enseignement primaire public à tous les enfants du pays ${ }^{6}$, il importait donc de multiplier ces établissements - et de les séculariser, le cas échéant -, de manière à constituer un contingent suffisant de maîtres. Á cet effet, l'importante loi du 9 août 1879 exigea de tous les départements qu'ils fussent pourvus de deux écoles normales (une pour chaque sexe) dans un délai de quatre $a^{7} s^{7}$. Si les conseils généraux avaient ainsi la responsabilité du cadre matériel, c'est à l'État qu'il revenait de recruter un nombreux personnel administratif et enseignant, dont les compétences devaient être plus étendues que par le passé, conformément au décret du 5 juin 1880 qui avait créé le titre de " professeur » d'école normale et organisé « une sorte d'agrégation de l'enseignement primaire $»^{8}$. La nécessité d'agir promptement, tout particulièrement en faveur des écoles normales de filles qui faisaient défaut presque partout, entraina la création d'une école normale «supérieure » d'institutrices (décret du 13 juillet 1880$)^{9}$, à laquelle on affecta bientôt une propriété à Yzeure, à deux kilomètres de Moulins.

3 La décision d'implanter si loin de la capitale un établissement d'instruction à vocation nationale avait un précédent : en 1865 déjà, le ministre Victor Duruy avait fondé à Cluny, au cœur de la Bourgogne, l'école normale d'enseignement secondaire spécial, mais tandis qu'un tel parti se justifiait par les importantes ressources agricoles, industrielles, commerciales et minières de la région, si profitables aux futurs élèves-maitres ${ }^{10}$, le choix du domaine d'Yzeure se fondait d'abord sur des considérations de politique locale. Ce site de dix hectares était une source de préoccupations depuis qu'une ordonnance royale l'avait gracieusement concédé au diocèse de Moulins (25 décembre 1822): contre toute attente, l'évêque y avait créé peu après un vaste collège, confié aux Jésuites, au grand dam du lycée de Moulins voisin et du conseil général de l'Allier qui n'eut de cesse désormais qu'il mette fin à cette situation préjudiciable ${ }^{11}$. Après moult péripéties, un décret du 31 juillet 1880 officialisa le changement d'affectation de la propriété ${ }^{12}$; le 11 août, le ministre accordait à l'évêque de Moulins « un délai d'un mois [...] pour rendre disponibles les locaux par lui occupés » et, dès le lendemain, il chargeait Charles Le Cœur de préparer l'installation de l'École dans lesdits locaux ${ }^{13}$.

Cette nomination s'était sans doute imposée spontanément à Jules Ferry : architecte du gouvernement depuis 1868 et déjà auteur du lycée de Bayonne (1874-1879), Le Cœur était en passe de devenir le plus sollicité des architectes du ministère de l'Instruction publique ${ }^{14}$. À l'été 1880, il étudiait des projets pour les lycées de Roanne et d'Aix-en-Provence, et s'apprêtait à mener des travaux au lycée Louis-le-Grand pour le compte de l'État ; surtout, il venait d'être choisi pour élever le lycée de Montluçon ( 5 janvier) et l'établissement thermal de Bourbon-l'Archambault (5 mai), tous deux situés précisément dans le département de l'Allier. Ses compétences reconnues en matière d'architecture scolaire et les liens qu'il avait déjà noués dans la région le désignaient donc tout particulièrement pour l'appropriation de la propriété d'Yzeure. Mais put-il seulement lever le plan des bâtiments existants? On peut en douter, car l'évêque de Moulins, peu conciliant, récusa l'injonction ministérielle et contre-attaqua sur le terrain judiciaire. En catastrophe, l'administration se mit donc en quête d'un nouveau site, susceptible d'accueillir la première promotion d'élèves en attendant le règlement de cette affaire. C'est ainsi que le 15 octobre 1880 un décret présidentiel autorisa le ministre « à acquérir de $\mathrm{M}$. [JosephAugustin] Pajou la propriété sise à Fontenay-aux-Roses (Seine), Grande-Rue, $\mathrm{n}^{\circ} 3$ [actuelle rue Boucicaut], pour servir à l'installation "provisoire" de l'École normale [sic] d'institutrices $»^{15}$. L'instigateur de cette soudaine transaction avait été manifestement un architecte du ministère, mais nous ignorons s'il s'agissait de Le Cœur lui-même, qui 
connaissait la propriété pour y avoir séjourné en famille à l'été 1873, en compagnie du jeune Auguste Renoir ${ }^{16}$, ou de Jean-Charles Laisné (auteur de la faculté de Pharmacie et du lycée Janson-de-Sailly), qui habitait une maison jouxtant la propriété Pajou ${ }^{17}$. Tous deux, sans doute, contribuèrent à convaincre le ministre.

Dans son rapport au président de la République en date du même jour, Ferry expliqua la combinaison qu'il avait élaborée: sitôt le contentieux réglé, l'établissement serait transféré à Yzeure et laisserait les locaux de Fontenay au cours pratique des salles d'asiles (ou école Pape-Carpantier), encore installé dans le Quartier latin mais menacé par une expropriation ${ }^{18}$. On sait ce qu'il advint de ce projet d'arrangement: l'école PapeCarpantier fut établie à Sceaux en 1882, puis à Versailles en 1886, avant d'être supprimée en 1891 ; quant à l'ENS, elle ne quittera Fontenay que 120 ans plus tard, en l'an 2000 !

\section{Une installation rudimentaire (1880)}

6 Le choix de la banlieue parisienne était moins insolite que celui de la province. Profitant du développement des transports ferroviaires, plusieurs établissements d'enseignement publics ou privés s'y étaient déjà fixés, et le gouvernement lui-même envisageait alors d'y fonder les futurs lycées d'internes de la capitale, suivant en cela un mouvement suscité par le collège Sainte-Barbe, dont l'annexe ("Sainte-Barbe-des-Champs ») avait été créée en $1852 \ldots$ à Fontenay-aux-Roses ${ }^{19}$. Perchée sur une colline, cette petite commune verdoyante et fleurie offrait un cadre aussi propice à la villégiature qu'à l'étude ; elle était directement reliée à Paris, à neuf kilomètres de là, par la ligne de chemin de fer de Sceaux et par une ligne de tramways, et le prix des terrains y était très abordable : celui de Pajou, qui couvrait un peu plus de 1,3 hectares, fut acquis par l'État pour 110000 francs, soit environ 8,46 francs par mètre carré2 ${ }^{20}$.

7 Organisé par Félix Pécaut, un ancien pasteur devenu inspecteur général de l'instruction publique (hors cadre) ${ }^{21}$, l'établissement commença son existence à la fin d'octobre 1880 , dans les locaux de l'école normale d'institutrices de la Seine, boulevard des Batignolles à Paris. Puis le 18 novembre, après que Le Cœur, "par des prodiges d'activité et d'ingéniosité, eut mis, dans l'espace de trois semaines, la vieille demeure en état de [les] recevoir $\aleph^{22}$, les dix-neuf élèves de la première promotion prirent enfin possession des locaux de Fontenay, «parmi une multitude d'ouvriers de tous corps de métier, occupés jour et nuit à clouer, peindre, entoiler des cloisons de planches, et à les sécher à grand renfort de braseros ${ }^{23}$.

8 La ci-devant propriété Pajou disposait d'un parc arboré à flanc de coteau, avec parterres gazonnés, massifs de fleurs, serre et potager. C'était son principal atout, car les deux longs corps de bâtiments qui s'élevaient de part et d'autre d'une cour d'entrée, au nord, dans la partie supérieure du terrain, étaient " disparates, délabrés, d'aspect sordide au dehors et lépreux au-dedans $\aleph^{24}$. Parce qu'il fallait agir vite et parce que l'installation ne devait être que " provisoire ", l'architecte n'exécuta d'abord que les aménagements les plus essentiels : il rafraîchit les deux ailes, les accommoda tant bien que mal à leur nouvel usage et les raccorda par une galerie vitrée (ill. 1). Au dire de plusieurs témoins, cette installation primitive était des plus rudimentaires ${ }^{25}$. 


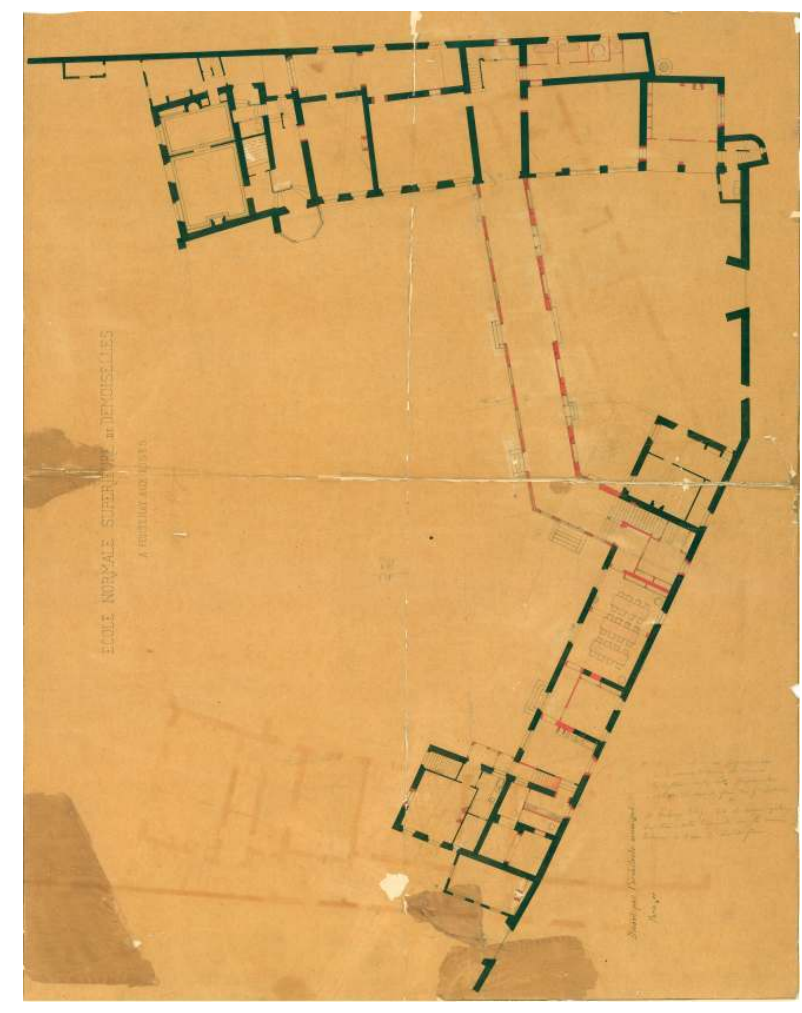

Automne 1880 : la première installation

CNAM/Cité de l'Architecture et du Patrimoine

9 L'aile droite, perpendiculaire à la rue, était spécialement affectée à l'administration et aux locaux d'enseignement: au rez-de-chaussée, on trouvait les deux salles de classes (pour les sciences et les lettres) et leurs annexes; au $1^{\mathrm{er}}$ étage, une unique salle d'étude servant aussi aux réunions, les cabinets de la directrice générale $\left(\mathrm{M}^{\mathrm{me}}\right.$ de Friedberg) et de la directrice des études ( $\mathrm{M}^{\text {me }}$ Chasteau), un petit dortoir et l'infirmerie; sous le comble, quelques chambres individuelles. L'aile gauche, alignée sur la rue, accueillait le logement du gardien, le réfectoire, les cuisines et sans doute le bureau de l'économe (rez-dechaussée), la salle de dessin et six nouvelles chambres ( $1^{\text {er }}$ étage). Conformément à ce que venait de recommander l'architecte Narjoux dans le premier ouvrage consacré à la construction des écoles normales ${ }^{26}$, les logements respectifs des deux directrices étaient tenus à l'écart des espaces fréquentés par les élèves: aménagés à l'extrémité de chaque aile, ils disposaient pareillement d'une entrée indépendante et embrassaient deux niveaux ${ }^{27}$. La galerie enfin, seule construction neuve, était un dispositif habile qui donnait sa cohésion à l'ensemble. Elle facilitait les circulations d'un bâtiment à l'autre, procurait à l'École un vestibule général et formait une aimable démarcation entre la cour et le parc. Face à l'entrée, un écran de pierres prolongeait les lignes du rez-de-chaussée de l'aile droite ; à l'arrière, le pan de bois se substituait à la maçonnerie (ill. 2). 
III. 2 : L'ancienne aile droite avant sa surélévation, la galerie vitrée de 1880 et le nouveau pavillon d'entrée

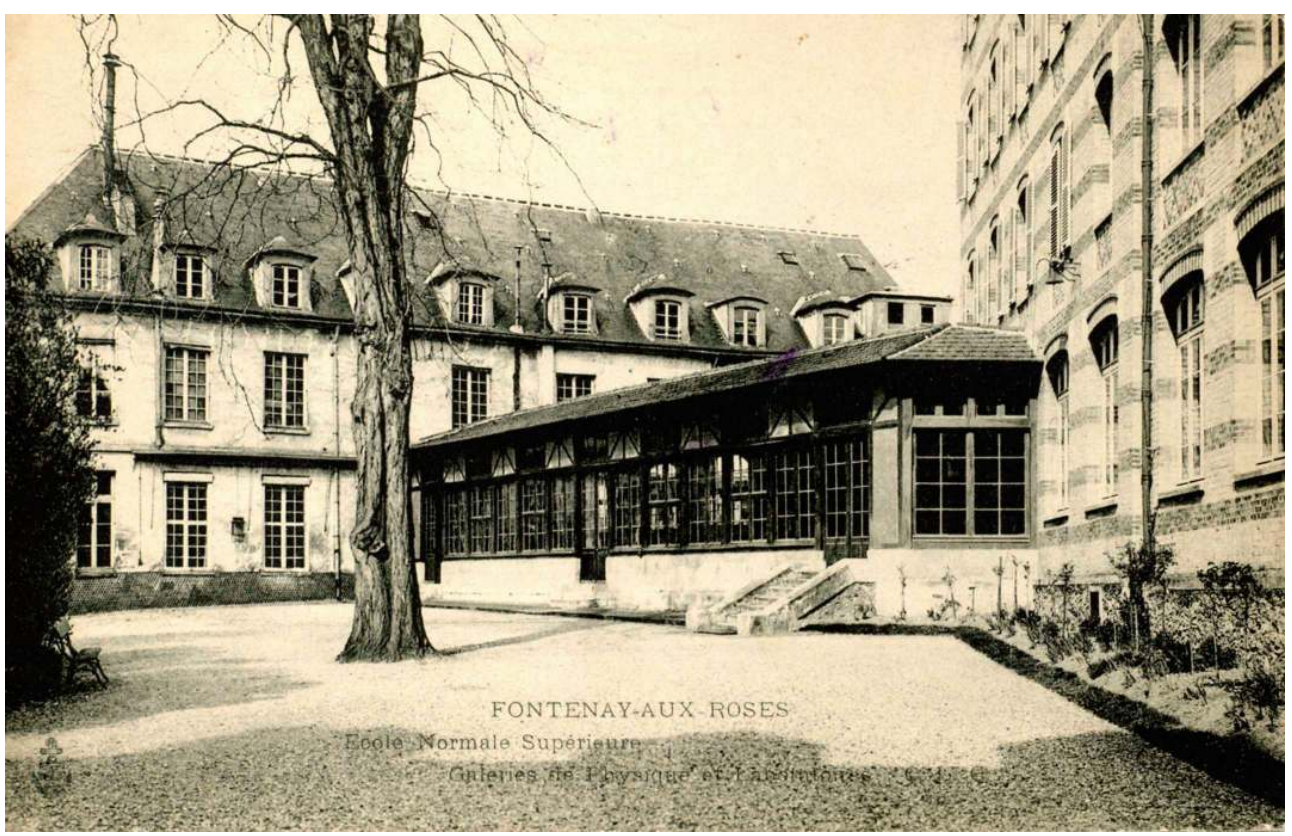

Carte postale ancienne, vers 1900

Cl. Marc Le Cœur

\section{L'impossible école normale d'application (1881-1884)}

Le premier projet d'agrandissement date de février 1881. Depuis l'automne précédent, le contexte avait beaucoup évolué : en obtenant la collaboration d'éminents professeurs attachés à l'Université, au Collège de France, au Muséum et aux lycées de la capitale ${ }^{28}$, Pécaut avait prouvé que « le voisinage de Paris était la première condition de succès » de l'établissement ${ }^{29}$; le déménagement à Yzeure n'était donc plus d'actualité. De plus, un second concours d'entrée, ouvert en janvier 1881, allait bientôt accroître l'effectif de l'École, ce qui rendait indispensables des services plus pérennes et mieux entendus. Il fut d'abord question d'un bâtiment isolé, à usage de classes, chambres individuelles et salles de dessin, qui devait être élevé à l'ouest du terrain et relié à l'aile droite par une nouvelle galerie de communication. Le 12 juillet, Le Cœur acheva un projet d'une tout autre ampleur.

11 Dès la fondation de l'établissement, l'administration aspirait à lui rattacher une école normale d'application, avec école primaire annexe, afin de confronter ses élèves aux réalités de leur future mission ${ }^{30}$. Les partisans du domaine d'Yzeure avaient d'ailleurs insisté sur la proximité de l'école normale d'institutrices de l'Allier. Celle de la Seine, en revanche, était très éloignée de Fontenay. Après l'abandon du projet de déménagement, il fallut donc envisager de remédier à cet inconvénient.

12 Le programme donné à Le Cœur était sans équivalent : il s'agissait de bâtir, sur un même site, trois écoles gigognes, de matérialiser en quelque sorte cette " cascade pédagogique » ${ }^{31}$ qui caractérisait désormais la logistique de l'enseignement primaire. Pour ce faire, l'architecte fractionna la propriété en trois parties distinctes et échelonna les 
établissements sur le coteau, comme pour signifier la subordination de chacun d'eux à celui qui le précédait (ill. 3).

\section{3 : Charles Le Cœur, Projet d'agrandissement avec construction d'une école normale annexe et} d'une école primaire

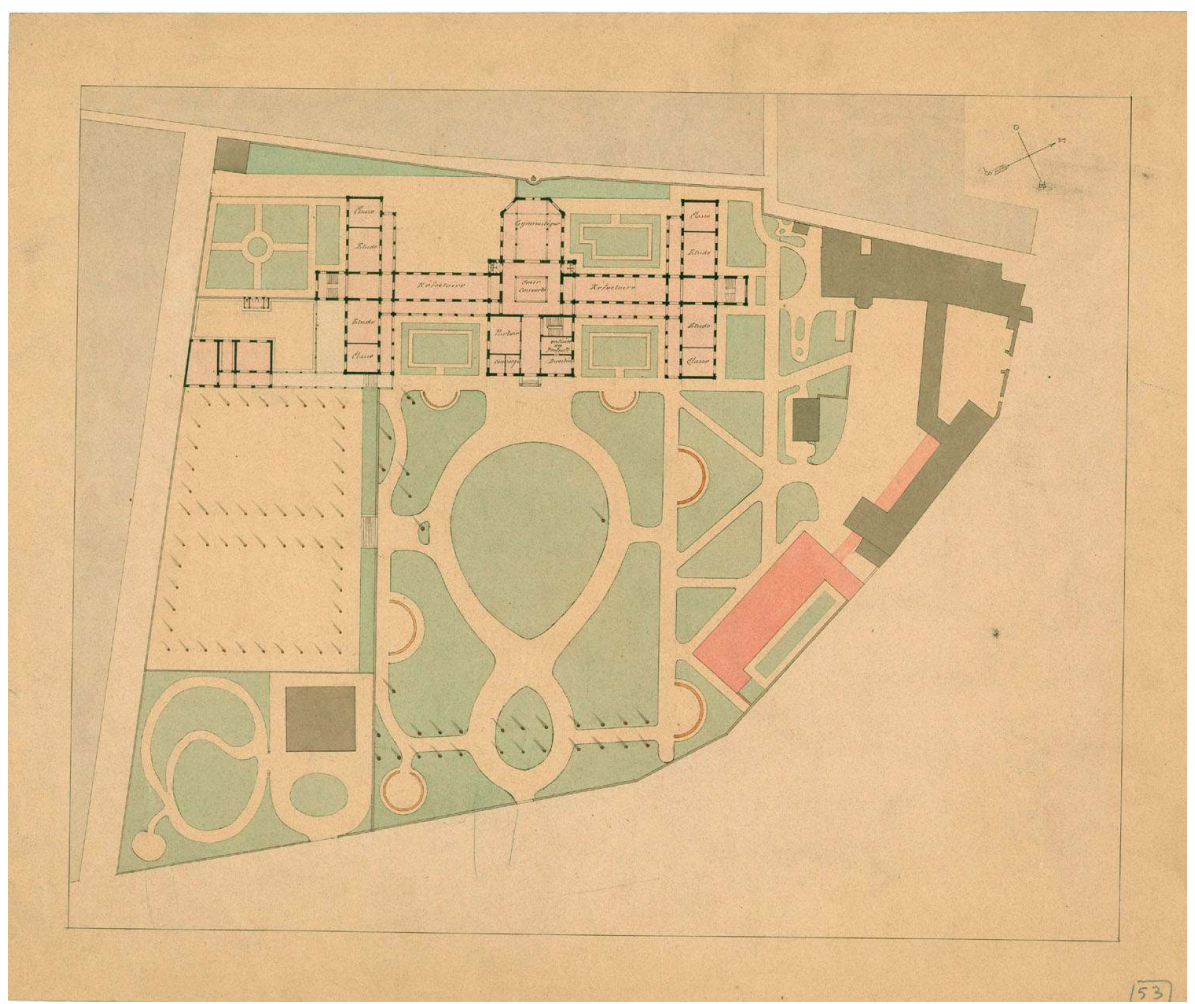

12 juillet 1881

CNAM/Cité de l'Architecture et du Patrimoine

En contre-haut, l'école normale supérieure était augmentée du bâtiment conçu quelques semaines plus tôt mais qui avait été déplacé en bordure de la Grande Rue, dans le prolongement de l'aile gauche ancienne; au fond de la partie centrale, à l'emplacement $\mathrm{du}$ potager, s'élevait une majestueuse école normale pouvant accueillir 120 pensionnaires; enfin, au niveau inférieur, on trouvait le logement des fonctionnaires (avec jardin privatif), la cour de récréation de l'école normale, puis une petite école primaire.

L'architecte avait prouvé la faisabilité de cet assemblage singulier. Pourtant, il dut tôt reprendre son étude... sur un autre site. Vers la fin de l'été, en effet, le ministère jeta son dévolu sur le lieu-dit «Les Mollins ", un terrain d'une superficie totale de plus de quatre hectares, qui s'étendait au sud-est de l'École, sur l'autre rive de l'avenue de Sceaux (actuelle avenue Lombart), et était encore morcelé en plusieurs dizaines d'étroites parcelles. La raison de ce nouveau revirement n'est pas connue, mais elle est peut-être liée à l'idée de réunir également à l'École de Fontenay l'école normale supérieure de l'enseignement secondaire féminin, fondée le 26 juillet $1881^{32}$. Jusqu'en mars 1884 , l'architecte proposa de multiples plans pour cet emplacement, souvent très aboutis. L'un d'eux intégrait dans une même composition l'école normale supérieure d'institutrices, l'école normale annexe, une école primaire et une école maternelle. Tous ces projets firent long feu, sans doute parce que le ministère ne put finalement se porter acquéreur 
de la totalité des parcelles qu'exigeait l'opération ${ }^{33}$. Devenus inutilisables, les terrains furent revendus avant $1893^{34}$ et, en dépit de ce que prescrivait toujours l'article $91 \mathrm{du}$ décret du 18 janvier $1887^{35}$, l'école d'application ne vit jamais le jour.

\section{La métamorphose de l'École (1882-1904)}

En marge de ces études stériles, Le Cœur ne négligeait pas l'ancienne propriété Pajou. Dans les premiers mois de 1882, les besoins s'étaient fait de nouveau très pressants : la prolifération des écoles normales d'institutrices dans les départements exigeait de former un nombre toujours croissant de professeurs et de directrices ${ }^{36}$, et Jules Ferry demandait que des cours fussent communs aux élèves de Fontenay et à celles de l'école PapeCarpantier qui venait d'être transportée à Sceaux ${ }^{37}$.

16 L'architecte commença par substituer un grand amphithéâtre de bois à la serre qui s'élevait à l'orée du parc, en vis-à-vis de la galerie vitrée. La communauté des Fontenaisiennes, que la salle de réunion de l'aile droite ne pouvait plus contenir, allait y suivre en particulier les célèbres conférences que Pécaut donnait au commencement de chaque journée ${ }^{38}$. Puis, développant le parti retenu dans son projet du 12 juillet 1881, Le Cœur chercha à étendre les bâtiments en bordure de la Grande Rue. Succédant à plusieurs études infructueuses, son plan daté du 6 août 1882 fut approuvé par le ministre Duvaux et mis à exécution : une partie de l'aile gauche fut amputée afin de créer une cour de service - étagée sur deux niveaux en raison de la déclivité du terrain -, à laquelle les fournisseurs pouvaient accéder depuis la rue; au-delà, il édifia une imposante construction en L, qu'une galerie angulaire reliait au reste de l'École (ill. 4) et où furent rassemblés les cabinets de Pécaut et de $\mathrm{M}^{\text {me }}$ de Friedberg, la bibliothèque, deux salles d'études et une salle de dessin, la cuisine, ses dépendances et le réfectoire, ainsi qu'une trentaine de chambres d'élèves et huit chambres de domestiques; l'extrémité orientale de l'aile sur rue était entièrement dévolue au logement de la directrice et de sa famille (ill. $5)$. 
III. 4 : Amorce de l'aile prolongeant le pavillon d'entrée (à gauche) et face nord du bâtiment conçu en 1882 , avec sa galerie couverte

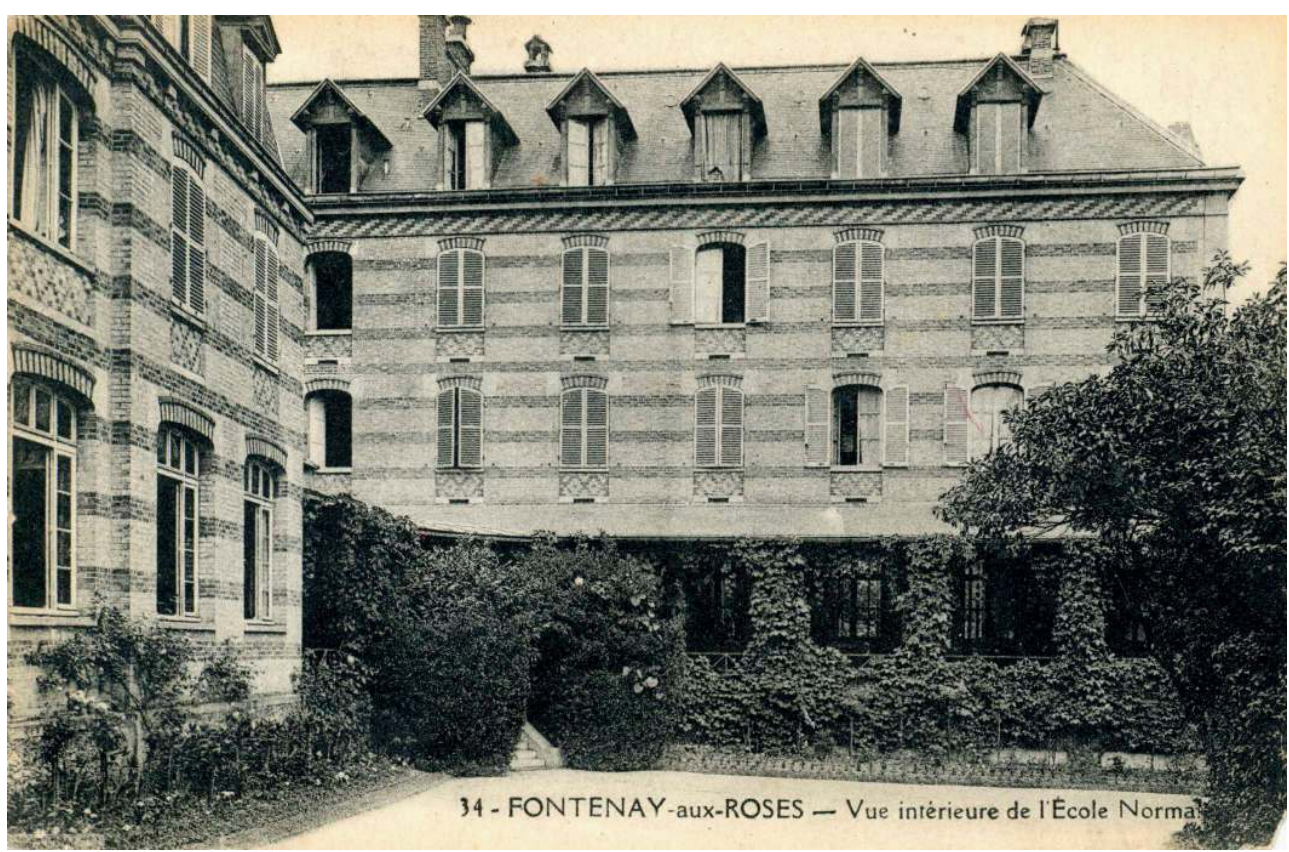

Carte postale ancienne, vers 1900

Cl. Marc Le Cœur.

III. 5 : Ensemble des bâtiments neufs de l'École, au long de la Grande Rue de Fontenay

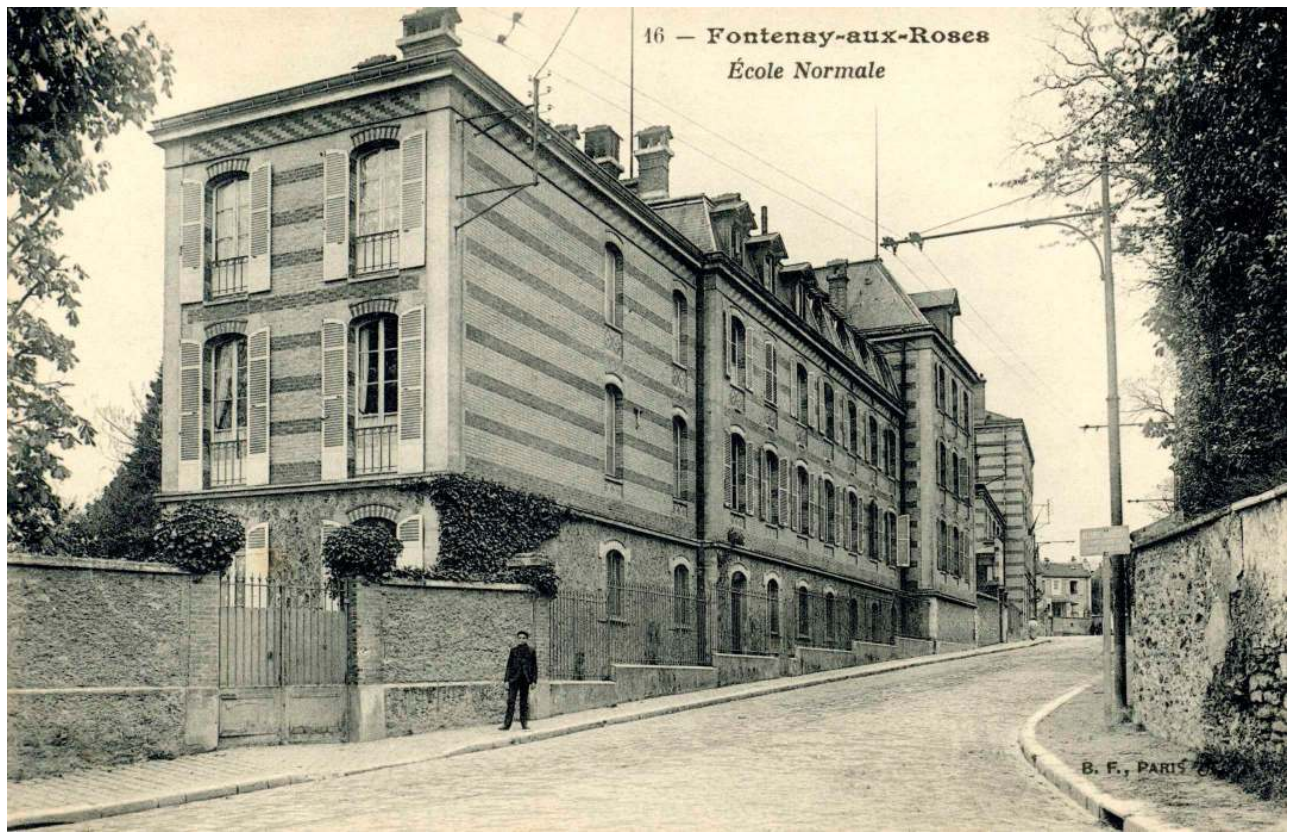

Carte postale ancienne, vers 1900

Cl. Marc Le Cœur.

17 Tandis que cette seconde campagne de travaux touchait à sa fin et alors que les projets d'expansion sur le terrain des Mollins paraissaient irrémédiablement compromis, l'administration se résolut à détruire ce qu'il restait de l'ancienne aile gauche. Un plan 
daté du 7 avril 1884 fut approuvé par le ministre Fallières et mis à exécution: à l'intersection des deux galeries (dont quelques travées furent intégrées à la nouvelle construction), l'architecte bâtit un véritable pavillon d'entrée, qu'une petite aile prolongeait latéralement jusqu'à la cour de service (ill. 6).

\section{6 : Charles Le Cœur, « École normale supérieure d'institutrices. Fontenay-aux-Roses}

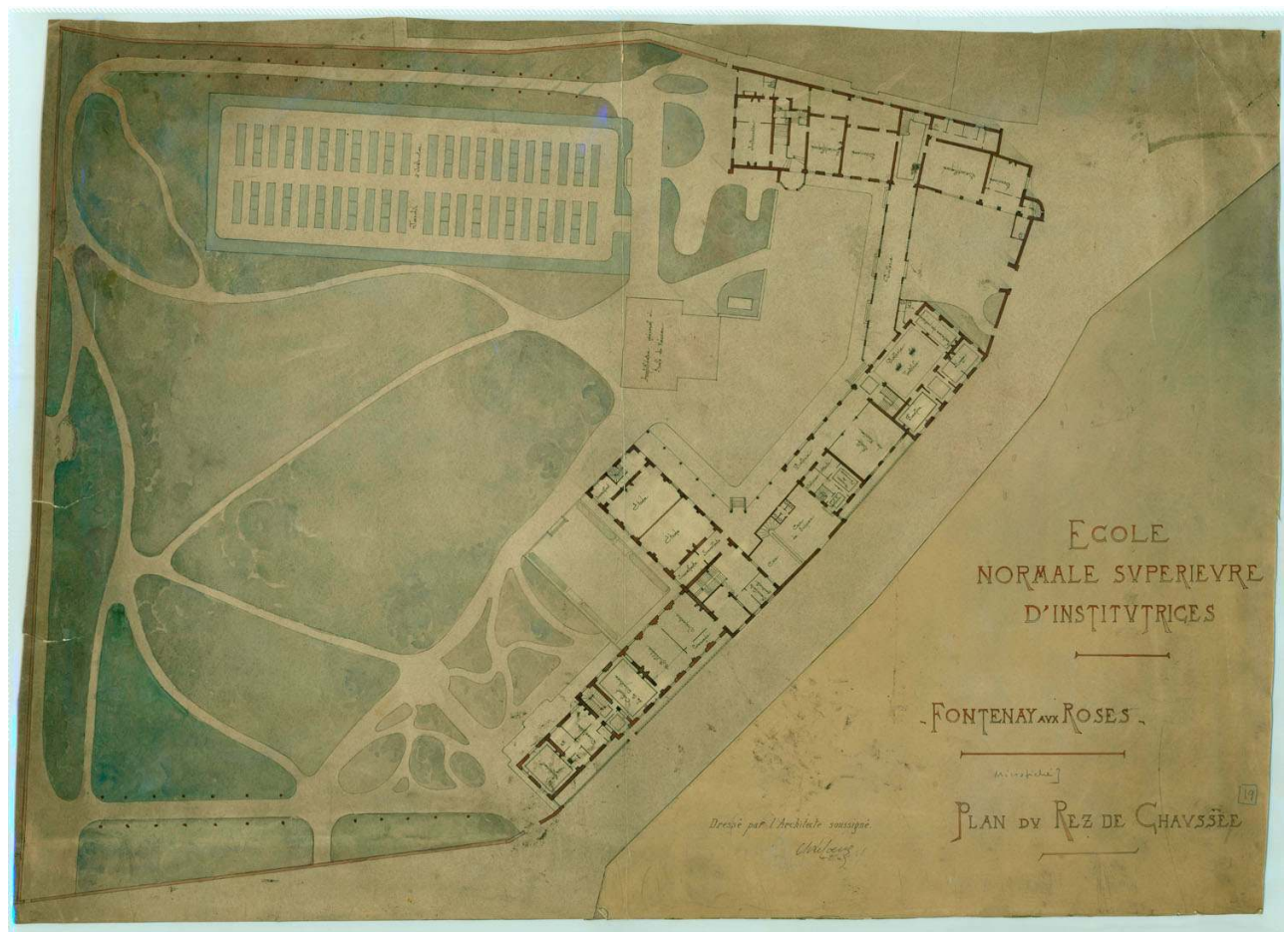

Plan du rez-de-chaussée », vers 1885 : l'établissement dans sa configuration définitive CNAM/Cité de l'Architecture et du Patrimoine

18 Le logement du concierge, un parloir et un vestiaire pour les professeurs y étaient réunis autour d'un large vestibule ; à leur suite, on trouvait une salle de classe et l'économat, et, à l'unique étage, des chambres d'élèves ainsi que la nouvelle salle de conférences. Dans le même temps, l'ancienne aile droite fut spécialement affectée à l'infirmerie et aux enseignements scientifiques, et la galerie vitrée de 1880, qui ne constituait plus le seuil de l'École, équipée pour les expériences de physique.

19 L'achèvement de la troisième campagne de travaux, à la fin de $1887^{39}$, ne marqua pas celui des transformations : dix ans plus tard, le cours normal des études venant d'être allongé d'un an ${ }^{40}$, il fallut étendre une nouvelle fois la capacité d'accueil de l'établissement. Plutôt que de construire un bâtiment neuf, Le Cœur se contenta de surélever ceux qui existaient : il exhaussa le pavillon d'entrée de deux étages (projet du 16 mars 1897), puis le bâtiment ancien d'un étage et demi (projet de juin 1903) ${ }^{41}$. Alors seulement l'École prit son apparence définitive, celle du moins qu'elle devait conserver pendant le demi-siècle suivant. 


\section{«L'esprit de Fontenay »}

20 considérablement réduit l'emploi de la pierre, supprimé un à un les éléments saillants ainsi que tous les artifices décoratifs, et limité les différents types de baies. Deux grands moyens lui permirent d'éviter la monotonie d'une masse architecturale uniforme. Pour dynamiser l'ensemble, il avait travaillé les volumes : il scinda le bâtiment en trois parties qu'il éleva chaque fois un peu plus en retrait de l'alignement sur rue et qu'il coiffa de toitures dont l'inclinaison s'accentuait graduellement. Pour égayer ses élévations, il avait travaillé la peau de l'édifice : au-dessus d'un soubassement de meulières, il adopta la brique de Bourgogne en parement, qu'il disposa en bandes continues, alternativement rouges et blanches. Á la même époque, d'autres architectes scolaires rayaient également leurs façades de briques, tels Baudot au lycée Lakanal (Sceaux, 1882-1885) ou Vaudremer dans ses lycées de jeunes filles (Michelet, à Montauban, 1883-1887 et Molière, à Paris, 1886-1888), mais nul n'osa pareil dépouillement. Á Fontenay, la planéité des murs et la régularité des assises n'étaient contrariées que par le léger renfoncement des allèges et le relief des arcs en anse de panier des fenêtres. La profusion des persiennes de bois, si inhabituelle dans un établissement d'enseignement, fût-il un internat, animait enfin les façades.

Ces multiples solutions, qui furent reprises dans les extensions ultérieures, donnaient à la demeure l'allure d'une grande et riante maison familiale. Cela traduisait parfaitement le caractère très libéral d'une école qui n'était rien moins qu'une « sorte de cloître studieux $»^{43}$ et où la discipline était des plus souples. Cela répondait également aux préoccupations de Pécaut, qui condamnait l'étalage de « ce faux luxe qui est l'une des plaies vives de notre société $»^{44}$ et 《le malin esprit bourgeois $»^{45}$, mais rejetait tout autant l'ascétisme et « l'esprit de mortification » ${ }^{46}$. Par la pondération de ses lignes et l'agrément de ses couleurs, l'architecture concourait donc à l'une des premières missions de l'établissement: l'éducation morale des jeunes femmes qui, à leur tour, allaient être bientôt appelées à former les institutrices. En cela, elle était un vecteur essentiel des idéaux laïques, républicains et, pour ainsi dire, féministes, que le gouvernement entendait répandre jusque dans les moindres communes de France.

Comme toutes celles et tous ceux qui furent associés, dès l'origine, à l'expérience de Fontenay, expérience unique dans l'histoire de notre pays, Le Cœur semble ne s'être jamais départi de son enthousiasme, en dépit du temps perdu à étudier vainement plus d'un projet. Le 16 avril 1896, alors que Pécaut s'apprêtait à prendre sa retraite, il lui confia :

J'aime la bâtisse comme vous le savez, mais j'aime encore et autant toutes les autres questions dont nous nous sommes entretenus depuis que j'ai eu le bonheur de vous rencontrer [...] dans le cabinet du cher Monsieur Buisson ${ }^{47}$.

Il ne pouvait exprimer plus simplement son adhésion aux valeurs prônées par Pécaut, ni mieux reconnaître combien leur collaboration avait été fructueuse. L'histoire de l'École de Fontenay fut, avant tout, une formidable aventure humaine. 


\section{NOTES}

1. Je tiens à remercier celles et ceux qui ont facilité la préparation de cet article : Armelle Le Goff (Archives nationales), Jean-Noël Luc (Université de Paris-IV), David Peyceré (cité de l'architecture et du patrimoine, Archives d'architecture du XX $\mathrm{XX}^{\mathrm{e}}$ siècle), et tout particulièrement Stéphanie Méchine (Archives du Rectorat de Paris).

Propos de Félix Pécaut, dans Notices sur les Écoles normales supérieures d'enseignement primaire de Fontenay-aux-Roses et de Saint-Cloud, Paris, Imprimerie nationale, 1889, p. 2.

2. Voir notamment Pascal Piéra, L'École normale d'institutrices du Puy-de-Dôme, Clermont-Ferrand, Un, Deux... Quatre Éditions, 2005, 151 p.

3. Exposition universelle internationale de 1900 à Paris. Rapports du jury international. Groupe I.Éducation et enseignement, $1^{\text {re }}$ partie, Paris, Imprimerie nationale, 1902, p. 163. Des établissements d'enseignement supérieur destinés spécifiquement aux femmes avaient été fondés en Angleterre et aux États-Unis avant 1880 (voir Marc Le Cœur, «Essai d'historiographie II. Des collèges médiévaux aux campus », Histoire de l'éducation, $n^{\circ} 102$, mai 2004, p. 59-60), mais aucun d'eux ne formait, comme l'École de Fontenay, des « institutrices d'institutrices ", selon l'heureuse formule de Ferdinand Buisson (F. Buisson, La Religion, la Morale et la Science : Leur Conflit dans l'Éducation contemporaine, Paris, Fischbacher, 1900, p. 151).

4. Les premières années de l'École ne sont documentées que par quelques documents épars, mais toutes les études de l'architecte sont parvenues jusqu'à nous (cité de l'architecture et du patrimoine, Archives d'architecture du XX $X^{\mathrm{e}}$ siècle (IFA), fonds Charles Le Cœur, IFA 148/01). Sur l'histoire de l'ENS, voir Yvonne Oulhiou, L'École normale supérieure de Fontenay-aux-Roses à travers le temps, 1880-1980, Fontenay-aux-Roses, 1981, 312 p., ill.

5. F. Buisson dir., Dictionnaire de pédagogie et d'instruction primaire [ci-après : DPIP], $1^{\text {re }}$ partie, tome II, Paris, Hachette, 1887, p. 2071.

6. La loi du 16 juin 1881 instituera sa gratuité et celle du 28 mars 1882, son caractère obligatoire et laïque.

7. Bulletin administratif de l'Instruction publique [ci-après : BAIP], 1879, p. 596-598.

8. DPIP, op. cit., p. 2448.

9. BAIP, 1880/I, p. 807. Il fallut attendre le 30 décembre 1882 pour que soit fondé, à Saint-Cloud, un établissement analogue pour les jeunes gens (BAIP, 1882/IV, p. 989-992).

10. DPIP, $1^{\text {re }}$ partie, tome I, Paris, Hachette, 1882, p. 411.

11. Sur « l'affaire d'Yzeure », voir Arch. nat., $\mathrm{F}^{17} 9400$.

12. BAIP, $1880 /$ I, p. 885-886.

13. Arch. Nat., $\mathrm{F}^{17} 9400$.

14. Sur sa carrière, voir Marc Le Cœur, Charles Le Cœur (1830-1906), architecte et premier amateur de Renoir, Paris, Réunion des musées nationaux, 1996, $111 \mathrm{p}$.

15. L'acte de vente sera signé le 6 décembre suivant, mais avec effet rétroactif au 15 octobre (Arch. nat., ET/CXVIII/1162)

16. Marc Le Cœur, «Le peintre, son premier modèle et ses premiers amateurs : l'histoire dont Renoir ne voulait pas parler ", Renoir, O Pintor da Vida, São Paulo, MASP, 2002, p. 214-215. 
17. Cette maison - dite plus tard «maison Laprade » - était au 7, Grande Rue. Elle fut acquise et annexée par l'ENS le 5 novembre 1951 (Fontainebleau, centre des archives contemporaines, 20020123/49).

18. Documents relatifs aux écoles normales supérieures de l'enseignement primaire, Paris, Imprimerie nationale, 1883, p. 12-13.

19. Marc Le Cœur, «Les lycées dans la ville: l'exemple parisien (1802-1914)», Histoire de l'éducation, $\mathrm{n}^{\circ}$ 90, mai 2001, p. 157-163.

20. Le terrain destiné au petit lycée Condorcet, au nord de la gare Saint-Lazare, sera acquis en 1881 pour 486,30 francs le mètre carré (ibid., p. 161).

21. Arrêté du 23 septembre 1880 (BAIP, 1880/II, p. 1196).

22. F. Pécaut, L'Éducation publique et la vie nationale, $2^{\mathrm{e}}$ éd., Paris, Hachette, 1904, p. 322.

23. Ibid.

24. Ibid.

25. Ibid., p.322-323; Bulletin de l'Association amicale des anciennes élèves de Fontenay-auxRoses [ci-après : $B A A F$ ], $\mathrm{n}^{\circ}$ 30, janvier 1904, p. 28, et $\mathrm{n}^{\circ}$ 33, octobre 1904, p. 162.

26. Félix Narjoux, Les Écoles normales primaires. Construction et installation, Paris, Delagrave/Morel et $C^{\text {ie }}, 1880$, p. 296.

27. Après la démission de $\mathrm{M}^{\text {me }}$ Chasteau, en mai 1881 (BAIP, 1881/I, p. 833), Pécaut reprit les fonctions qu'elle exerçait mais il n'habita jamais dans l'enceinte de l'École : l'administration louait pour sa famille et lui un pavillon non loin de là.

28. Arrêté du 3 novembre 1880, BAIP, 1880/II, p. 1448-1450.

29. Propos de Jules Ferry à la chambre des députés (9 juillet 1881), cité dans La Démocratie du Centre, $\mathrm{n}^{\circ} 157,17$ juillet 1881.

30. Le décret du 13 juillet 1880, art. 3, prescrivait que «l'école normale du département où [l'établissement] sera situé sera annexée comme école d'application audit établissement ».

31. Léo Claretie, L'Université Moderne, Paris, Delagrave, c. 1893, p. 23.

32. Le Cinquantenaire de l'École de Sèvres, 1881-1931, Paris, 1932, p. 124. Ce projet était encore évoqué après que le nouvel établissement eut été mis en service, le 12 décembre suivant, dans les locaux de l'ancienne manufacture de porcelaine à Sèvres (voir A. Darlu, « Félix Pécaut, directeur de l'École de Fontenay", Revue pédagogique, 15 février 1900, p. 116). Le Cœur fut l'architecte de l'École de Sèvres, comme de celle de Saint-Cloud.

33. En février 1884, neuf propriétaires contestèrent en justice leur expropriation (Fontenay-auxRoses, Archives municipales, $2 \mathrm{M}$ 101).

34. Y. Oulhiou, op. cit., p. 73.

35. BAIP, 1887/I, p. 116.

36. En 1889, l'effectif de l'École se monte, « depuis plusieurs années », à 71 élèves (Notices sur les Écoles normales supérieures..., op. cit., p. 3).

37. Circulaire du 28 juillet 1882, BAIP, 1882/III, p. 310.

38. Voir notamment Gabriel Compayré, Félix Pécaut et l'Éducation de la Conscience, Paris, Paul Mellottée, [1904], p. 53-55.

39. La nouvelle salle de conférences est «inaugurée» le 6 janvier 1888 (F. Pécaut, L'Éducation publique..., op. cit., p. 330-333).

40. Exposition universelle internationale de 1900..., op. cit.

41. Voir BAAF, articles cités.

42. E. Rivoalen, «Exposition universelle. L'architecture au Champ-de-Mars », La Construction moderne, 3 novembre 1900, p. 55.

43. F. Pécaut, dans $B A A F, \mathrm{n}^{\circ} 1$, novembre 1894, p. 2.

44. Ibid., p. 7. 
45. F. Pécaut, L'Éducation publique..., op. cit., p. 284.

46. G. Compayré, op. cit., p. 44 ; voir aussi Félix Pécaut, Quinze ans d'éducation (Notes écrites au jour le jour), Paris, Delagrave, 1902, p. 85.

47. Collection particulière. Ferdinand Buisson était le directeur de l'enseignement primaire depuis 1879 ; véritable initiateur de l'École, il quitta ses fonctions en même temps que Pécaut. Buisson, Pécaut et Le Cœur étaient tous trois protestants.

\section{RÉSUMÉS}

Fondée en 1880, l'École de Fontenay fut longtemps l'une des pièces maîtresses de l'enseignement primaire; c'est là, en outre, que l'enseignement universitaire français s'ouvrit aux femmes. Pourtant, en dépit de son rôle éminent, l'établissement ne s'incarna pas dans des constructions monumentales: des bâtiments vétustes abritèrent ses premières années, qui firent progressivement place à des bâtiments de briques l'assimilant à une confortable maison bourgeoise, ouverte sur un vaste parc. Son histoire matérielle fut marquée par la rencontre de deux personnalités protestantes : l'ancien pasteur Félix Pécaut, qui fut «l'âme » de l'École, et l'architecte Charles Le Cœur. Année après année, après moult projets avortés, le second sut traduire, dans les nouvelles constructions, les idéaux laïques et féministes que le premier entendait inculquer aux jeunes filles dont il avait la charge. Par la simplicité de ses lignes, l'école de Fontenay s'opposait à la nouvelle Sorbonne, élevée concomitamment. Toutes deux exprimaient cependant, chacune à leur manière, une certaine conception de la République.

Founded in 1880, the Ecole de Fontenay was long in one of the key positions in the system of primary education ; besides, it is also here the French higher education opened up to women.Yet, despite its significant part, the institution did not find expression in monumental buildings : it was housed in some ancient buildings during the first years, subsequently by brick buildings looking like a comfortable middle-class house, opened out onto a vast park. Its facilities history was marked by the meeting of two Protestant personalities : the old pastor, Félix Pécaut, who was the "spirit" of the school, and the architect Charles Le Coeur. Year after year, after many plans were wrecked, the second one successfully made the new buildings convey the secular and feminist ideals the first one meant to inculcate in the young girls he was responsible for. Built on simple lines, the Ecole de Fontenay contrasted with the new Sorbonne, erected at the same time. Yet, both of them convey in their own way a certain idea of the Republic.

Die 1880 in Fontenay gegründete die Lehrerbildungsanstalt (pädagogische Frauenhochschule) entwickelte die Grundlagen des Grundschulunterrichts und wurde außerdem die erste Universität in Frankreich., an der Frauen zugelassen waren. Die Hochschule wurde trotz ihrer wichtigen Bedeutung mit keinen großartigen Bauten ausgestattet: die schäbigen Bauten der Anfangsjahre wurden langsam durch Ziegelsteinbauten ersetzt, die den Anschein eines bequemen bürgerlichen Hauses mitten auf einer großen Parkanlage gaben. Die Geschichte der Schule wurde von dem Zusammenwirken zweier protestantischer Persönlichkeiten geprägt : des ehemaligen Pfarrers Félix Pécaut, «Seele » der Schule, mit dem Architekten Charles Le Cœur. Im Laufe der Jahre, nach mehreren aufgegebenen Projekten in seinen Bauten, gelang es dem zweiten, die laizistischen und feministischen Ideale, die der erste seinen Studentinnen beibringen wollte, architektonisch umzusetzen. Die Hochschule von Fontenay unterscheidet sich 
durch ihre einfachen Bauformen von den Bauten der neuen Sorbonne, die gleichzeitig gebaut wurde. Jedes Bauwerk auf seine Art repräsentiert eine bestimmte Vorstellung der Republik.

\section{AUTEUR}

\section{MARC LE CEEUR}

Marc Le Cœur, né en 1965, rédige actuellement une thèse sur l'histoire matérielle et architecturale des lycées parisiens (université de Paris-I). Il est l'auteur du catalogue d'une exposition présentée au musée d'Orsay, dont il avait été le commissaire : Charles Le Cour (1830-1906), architecte et premier amateur de Renoir, Paris, Réunion des musées nationaux, 1996. Il a également co-dirigé, avec Anne-Marie Châtelet, L'Architecture scolaire. Essai d'historiographie internationale, numéro spécial de la revue Histoire de l'éducation, $\mathrm{n}^{\circ} 102$, mai 2004. Il a en outre publié plus d'une quarantaine d'articles (dans Monuments historiques, Histoire de l'art, The Burlington Magazine, Histoire de l'éducation, etc.) et contributions à des ouvrages collectifs (Lycées, lycéens, lycéennes. Deux siècles d'histoire, sous la dir. de P. Caspard, J.-N. Luc et Ph. Savoie, Lyon, INRP, 2005 ; História de Educação, Arquitetura e Espaço Escolar, sous la dir. de M. L. A. Bencostta, São Paulo, Cortez, 2005 ; L'École de plein air. Une expérience pédagogique et architecturale dans l'Europe du $X X^{e}$ siècle, sous la dir. de A.-M. Châtelet, D. Lerch et J.-N. Luc, Paris, Éditions Recherches, 2003 ; Renoir, O Pintor da Vida, São Paulo, MASP, 2002 ; Le Patrimoine de l'éducation nationale, Charenton-lePont, Flohic, 1999 ; etc.). Parmi ses dernières publications, figurent « Lycées, 1802-1940. Prisons, casernes ou parcs ? ", Archi-Créé, n 324, février-mars 2006, pp. 32-41, et « Le parapluie, la gare et l'échiquier, ou les Halles revisitées ", Paris. Baltard. Les Halles, Nogent-sur-Marne, Communauté d'agglomération de la Vallée de la Marne, Acte IV, 2006, pp. 15-32. Adresse électronique : marc_le_coeur@yahoo.com 